\title{
Comparative Study on Sea Surface Temperature Estimation with Thermal Infrared Radiometer Data among Conventional MCSST, Split Window and Conjugate Gradient Based Methods
}

\author{
Kohei Arai \\ Graduate School of Science and Engineering \\ Saga University \\ Saga City, Japan
}

\begin{abstract}
Comparative study on Sea Surface Temperature: SST estimations among the conventional Multi-Channel Seat Surface Temperature: MCSST, split window method and the proposed Conjugate Gradient based method: CGM with Thermal Infrared Radiometer: TIR data through simulations is conducted. Utilizing the proposed linearized inversion of radiative transfer equation, SST can be estimated. SST estimation accuracy of the proposed method is compared to the conventional regression based method (Split Window and MCSST method). Through the simulation study, it is found that the proposed CGM based method is superior to the conventional regression based method.
\end{abstract}

Keywords-Sea Surface Temperature; radiative transfer equation; regression; conjugate gradient; MCSST; split window.

\section{INTRODUCTION}

Sea Surface Temperature: SST estimation with thermal infrared radiometer onboard satellites is well known and widely used in a variety of research fields, in particular climate changes, global warming, etc. SST estimation methods are proposed [1]-[4]. Most of these are based on regressive analysis and use several spectral bands in Thermal Infrared: TIR wavelength region. The most dominant atmospheric factor is precipitable water. Using the different wavelength TIR bands whose influences due to water vapor are different, it is possible to reduce the influence. The most popular method is Multi Channel Sea Surface Temperature: MCSST [5]. Also previously proposed SST estimation methods are summarized by I. Barton [6]. In the same time, comparative study among the previously proposed methods is well reported [7].

Based on radiative transfer equation, inversion based SST estimation method is proposed [8]. Nonlinear radiative transfer equation is linearized then optimum combination of wavelength regions are selected [9]. Other than that, Geographic Information System: GIS based neural network is proposed for SST estimation method [10]. In this paper, linearized inversion based SST estimation method is utilized. Conjugate gradient method is applied to solve the linearized radiative transfer equation.

The following section describes the proposed SST estimation method with some theoretical background followed by some experiments with the conventional regression based methods. Then conclusion is described together with some discussions.

\section{PROPOSED METHOD}

A. Theoretical Background on SST Estimation with Thermal Infrared Radiometer Data

Radiation from a blackbody with physical temperature of $\mathrm{T}$ is expressed in equation (1)

$$
B_{\nu}(T)=\frac{2 h c^{2}}{\lambda^{5}\left(\exp \left(\frac{h c}{\lambda k T}\right)-1\right)}\left[W \cdot c m^{-2} \cdot s r^{-1} \cdot \mu m^{-1}\right]
$$

Where

$$
\begin{aligned}
& \mathrm{k}: \text { Boltzman constant }[J / K] \\
& \mathrm{h}: \text { Plank constant }[J \cdot s] \\
& \mathrm{c}: \text { Light speed }[\mathrm{m} / \mathrm{s}] \\
& \lambda: \text { Wavelength at wave number } \nu
\end{aligned}
$$

The contribution from the atmosphere can be expressed as follows,

$$
\tau\left(\theta, z_{\infty}, z\right)=\exp \left\{-\int_{z}^{z_{\infty}} \frac{\rho(z) k(z)}{\cos (\theta)} d z\right\}
$$

Where

$$
\begin{aligned}
& \theta: \text { Observation zenith angle } \\
& \rho: \text { Density of atmospheric continuents } \\
& k: \text { Volume extinction coefficient }
\end{aligned}
$$

And

$$
\int_{z}^{z_{\infty}} \frac{\rho(z) k(z)}{\cos (\theta)} d z
$$

Is called as optical depth of the atmosphere.

\section{B. At Sensor Radiance of Thermal Infrared Radiometer}

For sea surface observation with TIR radiometers onboard remote sensing satellites, radiance includes three components, 
the contribution from sea surface, the contribution from the reflected radiance at sea surface and the contribution from the atmosphere.

$$
\begin{array}{r}
I(\theta)=\int_{\lambda_{1}}^{\lambda_{2}} \Phi(\lambda)\left[\left\{\epsilon_{\lambda} B_{\lambda}\left(T_{s}\right)+\left(1-\epsilon_{\lambda}\right)\right.\right. \\
\left.\int_{z_{s}}^{\infty} B_{\lambda}[T(z)] \frac{\partial \tau\left(\theta, z_{\infty}, z\right)}{\partial z} d z\right\} \cdot \tau\left(\theta, z_{\infty}, z_{s}\right) \\
\left.\quad+\int_{z_{s}}^{\infty} B_{\lambda}[T(z)] \frac{\partial \tau\left(\theta, z_{\infty}, z\right)}{\partial z} d z\right] d \lambda
\end{array}
$$

Where

$$
\begin{aligned}
T_{s} & : \text { Sea surface temperature }[\mathrm{K}] \\
\Phi & : \text { Spectral response function } \\
\epsilon & : \text { Emissivity } \\
\tau & : \text { Transparency }
\end{aligned}
$$

Spectral response function means spectral sensitivity function of spectral bands of TIR onboard satellites. In general, emissivity of sea surface in TIR wavelength region is almost 1. Therefore, the second term of the equation (3) can be neglected.

$$
\begin{aligned}
I(\theta)= & \int_{\lambda_{1}}^{\lambda_{2}} \Phi(\lambda)\left\{\epsilon_{\lambda} B_{\lambda}\left(T_{s}\right) \tau\left(\theta, z_{\infty}, z_{s}\right)\right. \\
& \left.+\int_{z_{s}}^{\infty} B_{\lambda}[T(z)] \frac{\partial \tau_{\lambda}\left(\theta, z_{\infty}, z\right)}{\partial z} d z\right\} d \lambda
\end{aligned}
$$

\section{Approximation of At Sensor Radiance}

Assuming spectral response function in the spectral wavelength region of spectral band is 1 , then the equation (4) and be rewritten as follows,

$$
\begin{aligned}
I(\theta) & =B_{\lambda}\left(T_{s}\right) \tau\left(\theta, z_{\infty}, z_{s}\right) . \\
& +\int_{z_{s}}^{\infty} B_{\lambda}[T(z)] \frac{\partial \tau_{\lambda}\left(\theta, z_{\infty}, z\right)}{\partial z} d z
\end{aligned}
$$

The second term of equation (5) can be approximated as follows,

$$
\int_{z_{s}}^{\infty} B_{i}[T(z)] \frac{\partial \tau_{i}\left(\theta, z_{\infty}, z\right)}{\partial z} d z=\left[1-\tau_{i}\left(\theta, z_{\infty}, z\right)\right] I_{a i}
$$

Where $I_{a i}$ denotes representative of spectral band $i$ of radiance. Atmospheric transparency can be rewritten as follows,

$$
\begin{gathered}
\tau_{i}(u, \theta)=c_{1 i} \exp \left[-1\left(c_{2 i}+c_{3 i} m\right) u^{c_{4 i}+c_{5 i} m}\right] \\
=c_{1 i} \exp \left[-\left(c_{2 i}+\frac{c_{3 i}}{\cos \theta}\right) u^{c_{4 i}+\frac{c_{5 i}}{\cos \theta}}\right] \\
m \approx 1 / \cos \theta
\end{gathered}
$$

Where $u$ denotes precipitable water while $m$ denotes slant length between sea surface and TIR instrument onboard satellites. In the TIR wavelength region, precipitable water is major absorbing continuants in the atmosphere. Through simulation studies with radiative transfer code of MODTRAN with six atmospheric models (Tropic, Mid. Latitude Summer, Mid. Latitude Winter, Sub Arctic Summer, Sub Arctic Winter and 1976 US Standard Atmosphere), the coefficients are obtained as shown in Table 1 .

TABLE I. COEFFICIENTS OF EQUATION (7) OBTAINED WITH MODTRAN OF ATMOSPHERIC SOFTWARE CODE

\begin{tabular}{|c||c|c|c|}
\hline & $c_{n 1}$ & $c_{n 2}$ & $c_{n 3}$ \\
\hline$c_{1 i}$ & 0.8507924 & 0.9356485 & 0.9253728 \\
\hline$c_{2 i}$ & -0.0754923 & -0.03505476 & -0.03752114 \\
\hline$c_{3 i}$ & 0.175898 & 0.08923810 & 0.1261287 \\
\hline$c_{4 i}$ & 1.451688 & 1.739096 & 1.679308 \\
\hline$c_{5 i}$ & -0.2339985 & -0.1563839 & -0.1293923 \\
\hline
\end{tabular}

Then $I_{a i}$ is calculated as follows,

$I_{a i}=F_{i}\left(I_{a k}\right)=A_{1 i}+A_{2 i} I_{a k}$

The coefficients in the equation (8) are calculated with MODTRAN in the same manner which is mentioned above. Table 2 shows the results.

TABLE II. COEFFICIENTS OF EQUATION (8) OBTAINED WITH MODTRAN OF ATMOSPHERIC SOFTWARE CODE

\begin{tabular}{|c||c|c|c|}
\hline & $A_{n 1}$ & $A_{n 2}$ & $A_{n 3}$ \\
\hline$A_{1 i}$ & $-0.88610 \times 10^{-6}$ & 0.0 & $0.75270 \times 10^{-6}$ \\
\hline$A_{2 i}$ & 0.62180 & 1.0 & 1.0590 \\
\hline
\end{tabular}

Consequently, radiance of spectral band $i$ can be expressed as follows,

$I_{i}=B_{i}\left[T_{s}\right] \tau_{i}(u, \theta)+\left[1-\tau_{i}(u, \theta)\right] F_{i}\left(I_{a k}\right)$

In order to avoid divergence of the solution, the following conditional equation is introduced.

$$
X=\frac{X_{\max }+X_{\min }}{2}+\frac{X_{\max }-X_{\min }}{\pi} \arctan \xi
$$

D. Iteration Method for SST, Precipitable Water, and Representative Radiance

The unknown factors are as follows,

$\boldsymbol{x}=\left(T_{s}, u, I_{a k}\right)$

Namely, sea surface temperature, precipitable water, and representative radiance. The following cost function is introduced,

$$
J(\boldsymbol{x})=\sum_{i=1}^{3}\left(I_{i}-\hat{I}_{i}\right)^{2}
$$

Then iteration is stopped when the cost function is below the designated value,

$J(\boldsymbol{x}) \leq \varepsilon$

Radiance of the spectral band $i$ can be rewritten as follows,

$$
\begin{aligned}
I_{i} & =\frac{c 1 c_{1 i}}{\lambda^{3} \exp \left(\frac{c 2}{\lambda T_{s}}\right)-1} \exp \left\{-\left(c_{2 i}+\frac{c 3 i}{\cos \theta}\right) u^{c_{4 i}+\frac{c 5 i}{\cos \theta}}\right\} \\
& +\left[1-c_{1 i} \exp \left\{-\left(c_{2 i}+\frac{c_{3 i}}{\cos \theta}\right) u^{c_{4 i}+\frac{c 5 i}{\cos \theta}}\right]\left(C_{1 i}+C_{2 i} I_{a k}\right)\right.
\end{aligned}
$$


Then the following updating equation is introduced,

$\boldsymbol{x}^{(n+1)}=\boldsymbol{x}^{(n)}+\beta^{(n)} A^{(n)} \nabla J\left[\boldsymbol{x}^{(x)}\right]$

It is rewritten in matrix and vector as follows,

$$
\begin{aligned}
& \left(\begin{array}{c}
T_{s} \\
u \\
I_{a k}
\end{array}\right)^{(n+1)}=\left(\begin{array}{c}
T_{s} \\
u \\
I_{a k}
\end{array}\right)^{(n)} \\
& +\beta^{(n)}\left(\begin{array}{ccc}
\frac{\partial^{2} J}{\partial T_{s}^{2}} & \frac{\partial^{2} J}{\partial T_{s} \partial u} & \frac{\partial^{2} J}{\partial T_{s} \partial I_{a k}} \\
\frac{\partial^{2} J}{\partial u \partial T_{s}} & \frac{\partial^{2} J}{\partial u^{2}} & \frac{\partial^{2} J}{\partial u \partial I_{a k}} \\
\frac{\partial^{2} J}{\partial I_{a k} \partial T_{s}} & \frac{\partial^{2} J}{\partial I_{a k} \partial u} & \frac{\partial^{2} J}{\partial I_{a k}^{2}}
\end{array}\right)^{-1}\left(\begin{array}{c}
\frac{\partial^{2} J}{\partial T_{s}} \\
\frac{\partial^{2} J}{\partial u} \\
\frac{\partial^{2} J}{\partial I_{a k}}
\end{array}\right)^{(n)}
\end{aligned}
$$

Where

$$
\beta^{(n)}=1 / 2^{n}
$$

E. Conjugate Gradient Method

In general,

$$
\nabla f(x)=\left(\frac{\partial f(\boldsymbol{x})}{\partial x_{1}}, \frac{\partial f(\boldsymbol{x})}{\partial x_{2}}, \cdots, \frac{\partial f(\boldsymbol{x})}{\partial x_{n}}\right)^{\tau}
$$

And

$$
\nabla^{2} f(x)=\left(\begin{array}{cccc}
\frac{\partial^{2} f(x)}{\partial x_{1}^{2}} & \frac{\partial^{2} f(x)}{\partial x_{1} \partial x_{2}} & \cdots & \frac{\partial^{2} f(x)}{\partial x_{1} \partial x_{n}} \\
\frac{\partial^{2} f(x)}{\partial x_{1} \partial x_{1}} & \frac{\partial^{2} f(x)}{\partial x_{2}^{2}} & \cdots & \frac{\partial^{2} f(x)}{\partial x_{2} \partial x_{n}} \\
\vdots & \vdots & \ddots & \vdots \\
\frac{\partial^{2} f(x)}{\partial x_{n} \partial x_{1}} & \frac{\partial^{2} f(x)}{\partial x_{n} \partial x_{2}} & \cdots & \frac{\partial^{2} f(x)}{\partial x_{n}^{2}}
\end{array}\right)
$$

Therefore, the cost function can be rewritten as follows, $J=f(x), H_{n}=\nabla^{2} f(s)$

Where

$$
H_{n}=\left(\begin{array}{ccc}
\frac{\partial^{2} J}{\partial T_{s}^{2}} & \frac{\partial^{2} J}{\partial T_{s} \partial u} & \frac{\partial^{2} J}{\partial T_{s} \partial I_{a k}} \\
\frac{\partial^{2} J}{\partial u \partial T_{s}} & \frac{\partial^{2} J}{\partial u^{2}} & \frac{\partial^{2} J}{\partial u \partial I_{a k}} \\
\frac{\partial^{2} J}{\partial I_{a k} \partial T_{s}} & \frac{\partial^{2} J}{\partial I_{a k} \partial u} & \frac{\partial^{2} J}{\partial I_{a k}}
\end{array}\right)
$$

is called Hessian or Hesse matrix.

Equation (1) can be rewritten as follows,

$$
\begin{gathered}
B_{\nu}\left(T_{s}\right)=\frac{c_{i}}{\exp \left(\frac{c_{0} i}{T_{s}}\right)-1} \\
B_{\nu}(T)=\frac{c 1}{\lambda^{3}\left(\exp \left(\frac{c 2}{\lambda T}\right)-1\right)}
\end{gathered}
$$

where $c_{i}: 2 h c^{2} / \lambda_{i}^{5}$

$c_{0 i}: c h / \lambda_{i} k$

$c 1: 2 h c=1.191126^{-12}$

$c 2: c h / k=1.43889$

Then the unknown variables are estimated through iterations.

\section{F. Estimation of Hessian}

The first derivatives of the cost function are expressed as follows,

$$
\begin{gathered}
\frac{\partial J}{\partial T_{s}}=-2 \sum_{i=1}^{3}\left(I_{i}-\hat{I}_{i}\right) \frac{\partial \hat{I}}{\partial T_{s}} \\
\frac{\partial J}{\partial u}=-2 \sum_{i=1}^{3}\left(I_{i}-\hat{I}_{i}\right) \frac{\partial \hat{I}}{\partial u} \\
\frac{\partial J}{\partial I_{a k}}=-2 \sum_{i=1}^{3}\left(I_{i}-\hat{I}_{i}\right) \frac{\partial \hat{I}}{\partial I_{a k}}
\end{gathered}
$$

Also the second derivatives are represented as follows,

$$
\begin{gathered}
\frac{\partial^{2} J}{\partial T_{s}^{2}}=2 \sum_{i=1}^{3}\left\{\frac{\partial \hat{I}_{i}}{\partial T_{s}} \frac{\partial \hat{I}_{i}}{\partial T_{s}}-\left(I_{i}-\hat{I}_{i}\right) \frac{\partial^{2} \hat{I}_{i}}{\partial T_{s}^{2}}\right\} \\
\frac{\partial^{2} J}{\partial T_{s} \partial u}=2 \sum_{i=1}^{3}\left\{\frac{\partial \hat{I}_{i}}{\partial u} \frac{\partial \hat{I}_{i}}{\partial T_{s}}-\left(I_{i}-\hat{I}_{i}\right) \frac{\partial^{2} \hat{I}_{i}}{\partial T_{s} \partial u}\right\}
\end{gathered}
$$$$
\frac{\partial^{2} J}{\partial T_{s} \partial I_{a k}}=2 \sum_{i=1}^{3}\left\{\frac{\partial \hat{I}_{i}}{\partial I_{a k}} \frac{\partial \hat{I}_{i}}{\partial T_{s}}-\left(I_{i}-\hat{I}_{i}\right) \frac{\partial^{2} \hat{I}_{i}}{\partial T_{s} \partial I_{a k}}\right\}
$$$$
\frac{\partial^{2} J}{\partial u \partial T_{s}}=2 \sum_{i=1}^{3}\left\{\frac{\partial \hat{I}_{i}}{\partial T_{s}} \frac{\partial \hat{I}_{i}}{\partial u}-\left(I_{i}-\hat{I}_{i}\right) \frac{\partial^{2} \hat{I}_{i}}{\partial u \partial T_{s}}\right\}
$$$$
\frac{\partial^{2} J}{\partial u^{2}}=2 \sum_{i=1}^{3}\left\{\frac{\partial \hat{I}_{i}}{\partial u} \frac{\partial \hat{I}_{i}}{\partial u}-\left(I_{i}-\hat{I}_{i}\right) \frac{\partial^{2} \hat{I}_{i}}{\partial u^{2}}\right\}
$$$$
\frac{\partial^{2} J}{\partial u \partial I_{a k}}=2 \sum_{i=1}^{3}\left\{\frac{\partial \hat{I}_{i}}{\partial I_{a k}} \frac{\partial \hat{I}_{i}}{\partial u}-\left(I_{i}-\hat{I}_{i}\right) \frac{\partial^{2} \hat{I}_{i}}{\partial u \partial I_{a k}}\right\}
$$$$
\frac{\partial^{2} J}{\partial T_{s} \partial T_{s}}=2 \sum_{i=1}^{3}\left\{\frac{\partial \bar{I}_{i}}{\partial T_{s}} \frac{\partial \hat{I}_{i}}{\partial I_{a k}}-\left(I_{i}-\hat{I}_{i}\right) \frac{\partial^{2} \bar{I}_{i}}{\partial I_{a k} \partial T_{s}}\right\}
$$$$
\frac{\partial^{2} J}{\partial I_{a k} \partial u}=2 \sum_{i=1}^{3}\left\{\frac{\partial \hat{I}_{i}}{\partial u} \frac{\partial \hat{I}_{i}}{\partial I_{a k}}-\left(I_{i}-\hat{I}_{i}\right) \frac{\partial^{2} \hat{I}_{i}}{\partial I_{a k} \partial u}\right\}
$$$$
\frac{\partial^{2} J}{\partial I_{a k}^{2}}=2 \sum_{i=1}^{3}\left\{\frac{\partial \hat{I}_{i}}{\partial I_{a k}} \frac{\partial \hat{I}_{i}}{\partial I_{a k}}-\left(I_{i}-\hat{I}_{i}\right) \frac{\partial^{2} \hat{I}_{i}}{\partial I_{a k}^{2}}\right\}
$$

The first derivatives of radiance are expressed as follows, 


$$
\begin{gathered}
\frac{\partial I_{i}}{\partial T_{s}}=\frac{c_{i} c_{0 i} c_{1 i}}{T_{s}^{2}\left\{\exp \left(\frac{c_{0 i}}{T_{s}}\right)-1\right\}^{2}} \exp \left\{\frac{c_{0 i}}{T_{s}}-\alpha_{1} u^{\alpha_{2}}\right\} \\
\frac{\partial I_{i}}{\partial u}=c_{1 i} \alpha_{1} \alpha_{2} \alpha_{3} u^{\alpha_{2}-1}\left\{\left(C_{1 i}+C_{2 i} I_{a k}\right)-\frac{c_{i}}{\exp \left(\frac{c_{0 i}}{T_{s}}\right)-1}\right\} \\
\frac{\partial I_{i}}{\partial I_{a k}}=C_{2 i}\left\{1-c_{1 i} \exp \left(-\alpha_{1} u^{\alpha_{2}}\right)\right\}
\end{gathered}
$$

Also the second derivatives of radiance is represented as follows,

$$
\begin{aligned}
& \frac{\partial^{2} I_{i}}{\partial T_{s}}=\left[c_{i} c_{0 i} c_{1 i} \exp \left\{-\alpha_{1} u^{\alpha_{2}}\right\}\right] \\
& \left\{\frac{-c_{0 i} e^{\frac{c_{0 i}}{T_{s}}}-2 T_{s} e^{\frac{c_{0 i}}{T_{s}}}}{T^{4}\left(e^{\frac{c_{01}}{T_{s}}}-1\right)^{2}}+\frac{2 c_{0 i} e^{\frac{c_{0 i}}{T_{s}}}}{T^{4}\left(e^{\frac{c_{01}}{T_{s}}}-1\right)^{3}}\right\} \\
& \frac{\partial^{2} I_{i}}{\partial T_{s} \partial u}=-\frac{c_{i} c_{0 i} c_{1 i}}{T_{s}^{2}\left\{\exp \left(\frac{c_{0 i}}{T_{s}}\right\}^{2}\right.} \exp \left\{\frac{c_{0 i}}{T_{s}}-\alpha_{1} u^{\alpha_{2}}\right\} \alpha_{1} \alpha_{2} u^{\alpha_{2}-1} \\
& \frac{\partial I_{i}}{\partial T_{s} \partial I_{a k}}=0 \\
& \frac{\partial^{2} I_{i}}{\partial u \partial T_{s}}=-\frac{c_{i} c_{0 i} c_{1 i}}{T_{s}^{2}\left\{\exp \left(\frac{c_{0 i}}{T_{s}}\right)-1\right\}^{2}} \alpha_{1} \alpha_{2} \\
& \exp \left\{\frac{c_{0 i}}{T_{s}}-\alpha_{1} u^{\alpha_{2}}\right\} u^{\alpha_{2}-1} \\
& \frac{\partial^{2} I_{i}}{\partial u^{2}}=c_{1 i} \alpha_{1} \alpha_{2}\left\{\left(C_{1 i}+C_{2 i} I_{a k}\right)-\frac{c_{i}}{\exp \left(\frac{c_{0 i}}{T_{s}}\right)-1}\right\} \\
& \frac{\partial^{2} I_{i}}{\partial u \partial I_{a k}}=c_{1 i} C_{2 i} \alpha_{1} \alpha_{2} \alpha_{3} u^{\alpha_{2}-1} \\
& \frac{\partial^{2} I_{i}}{\partial I_{a k} \partial T_{s}}=0 \\
& \frac{\partial^{2} I_{i}}{\partial I_{a k} \partial u}=c_{1 i} C_{2 i} \alpha_{1} \alpha_{2} \alpha_{3} u^{\alpha_{2}-1} \\
& \frac{\partial^{2} I_{i}}{\partial I_{a k}^{2}}=0
\end{aligned}
$$

where

$$
\begin{gathered}
\alpha_{1}=c_{2 i}+\frac{c_{3 i}}{\cos \theta} \\
\alpha_{2}=c_{4 i}+\frac{c_{5 i}}{\cos \theta} \\
\alpha_{3}=\exp \left\{-\alpha_{1} u^{\alpha_{2}}\right\}
\end{gathered}
$$

\section{G. Conventional Regression Based Method of Split Window}

Split window method is based on regression with some training dataset which consists of truth data of SST and TIR spectral band data.
Namely, once regression analysis is made, then SST can be estimated with TIR spectral band data $\left(\mathrm{TIR}_{\mathrm{i}}\right)$. Regression analysis uses the following regressive equation,

$T_{S}=\sum_{i} c_{i} T I R_{i}$

Where $c_{i}$ denotes regressive coefficients for spectral band $i$.

\section{H. Conventional MCSST}

Multi Channel Sea Surface Temperature: MCSST is the method for SST estimation with NOAA/AVHRR (National Oceanic and Atmospheric Administration / Advanced Very High Resolution Radiometer) data. MCSST is based on split window method. AVHRR consist five channels, two channels in visible band, one channel in shortwave infrared band, and two channels in thermal infrared bands (Band 4 and 5). In general, MCSST is expressed in equation (28).

$$
\begin{aligned}
T_{s} & =A \cdot T B_{4}+B\left(T B_{4}-T B_{5}\right)+C\left(T B_{4}-T B_{5}\right)(\sec (\theta)-1) \\
& +D(\sec (\theta)-1)+E
\end{aligned}
$$

where

$$
\begin{aligned}
T_{s} & : \text { Sea Surface Temperature } \\
T B_{i} & : \text { Brightness Temperature of Band } \mathrm{i} \\
\theta & : \text { Observation Zenith Angle }
\end{aligned}
$$$$
A, B, C, D, E: \text { Regression Coefficients }
$$

In accordance with Kidwell, 1991, MCSST is expressed as follows,

$$
\begin{array}{rlr}
T_{\mathrm{S}}= & 0.9731 T_{4}+2.6353\left(T_{4}-T_{5}\right)-265.4789 & \text { for daytime, } \\
T_{\mathrm{S}}= & 0.9994 T_{4}+2.7057\left(T_{4}-T_{5}\right)-0.27\left(T_{4}-T_{5}\right)\left(\sec \theta_{z}-1\right) \\
& +0.73\left(\sec \theta_{z}-1\right)-273.0323 \quad \text { for nighttime, }
\end{array}
$$

where $\mathrm{T}_{\mathrm{S}}$ is given in ${ }^{\circ} \mathrm{C}$ and $\mathrm{T}_{4}$ and $\mathrm{T}_{5}$ are in Kelvin. The RMSE and the bias is $1.03^{\circ} \mathrm{C}$ and $-0.51^{\circ} \mathrm{C}$ for daytime, and $0.73^{\circ} \mathrm{C}$ and $0.19^{\circ} \mathrm{C}$ for nighttime, respectively.

\section{EXPERIMENT}

\section{A. Experimental Conditions}

The following parameters are set for the experiments with MODTRAN obtaining at sensor radiance of spectral TIR band data.

Atmospheric Model: Tropic, Mid.Latitude Summer,

Mid.Latitude Winter, SubArctic Summer, SubArctic

Winter, 1976 US Standard Atmosphere

Constraint: $\pm 2[\mathrm{~K}]$

Meteorọlogical Range: $\pm 0 \%, \pm 10 \%, \pm 20 \%$

Relative Humidity: Default x1.0,x.1.1,x1.2,x0.9,x0.8

Air-Temperature: Default $\pm 0, \pm 3[\mathrm{~K}]$

Sea surface temperature: Default $\pm \mathbf{0}, \pm 3[\mathrm{~K}]$

Wind speed: $3.5,7.0,14.0[\mathrm{~m} / \mathrm{s}]$

Aerosol Model: Navy Maritim,Maritim,Tropospheric,Desert Observation Zenith Angle: $0,30,60[\mathrm{deg}]$ 
Then SST is estimated with the proposed method and the conventional split window method using the calculated at sensor radiance.

\section{B. Example of Solution Behavior}

RMSE is estimated with a variety of precipitable water and representative radiance of TIR band. One of the examples of solution behavior is illustrated in Figure 1.

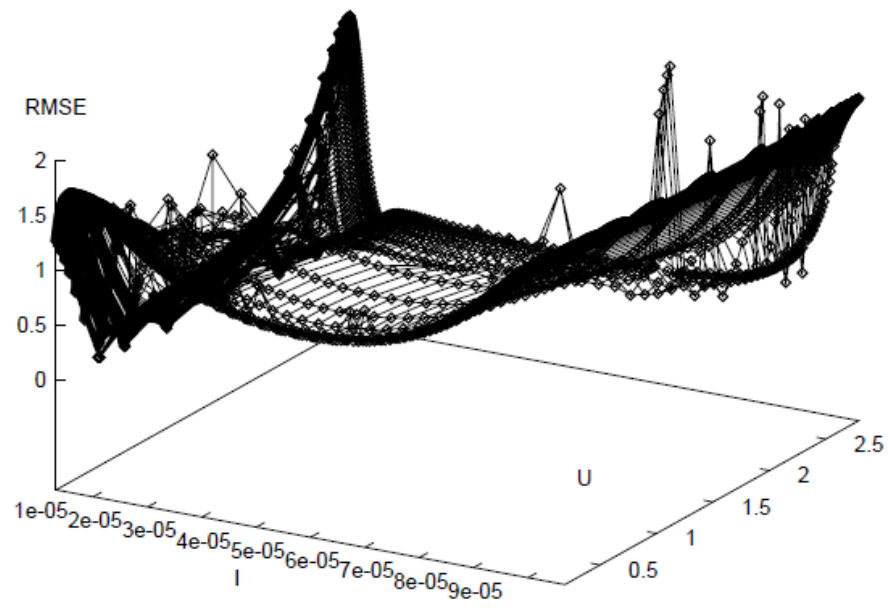

Fig.1. Example of solution behavior in solution space

In general, RMSE (SST estimation error) is proportional to the precipitable water. On the other hands, the relation between representative radiance of TIR band and RMSE shows complicated characteristics, in particular for small representative radiance regions. This implies that it is not easy to find the global optimum in the solution space. In other words, it is easy to fall in one solution of local minima.

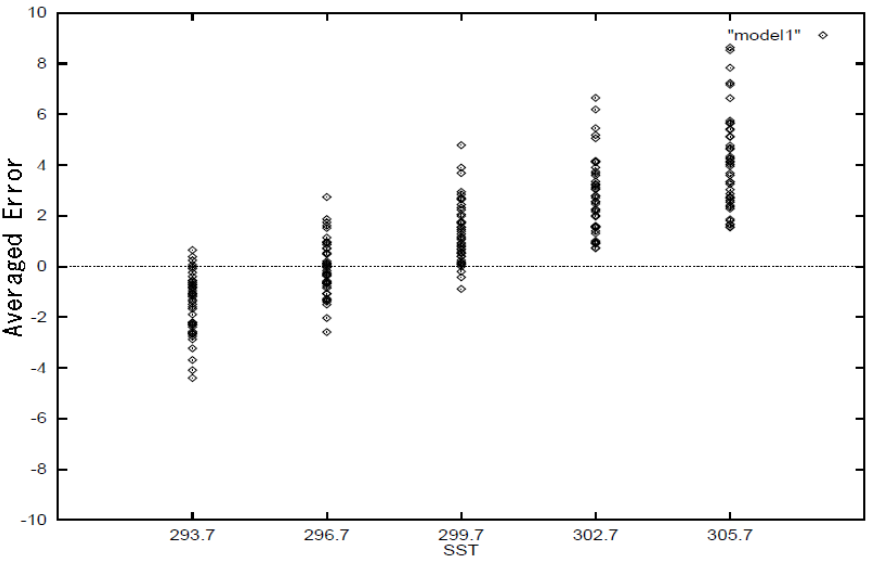

(a)MCSST

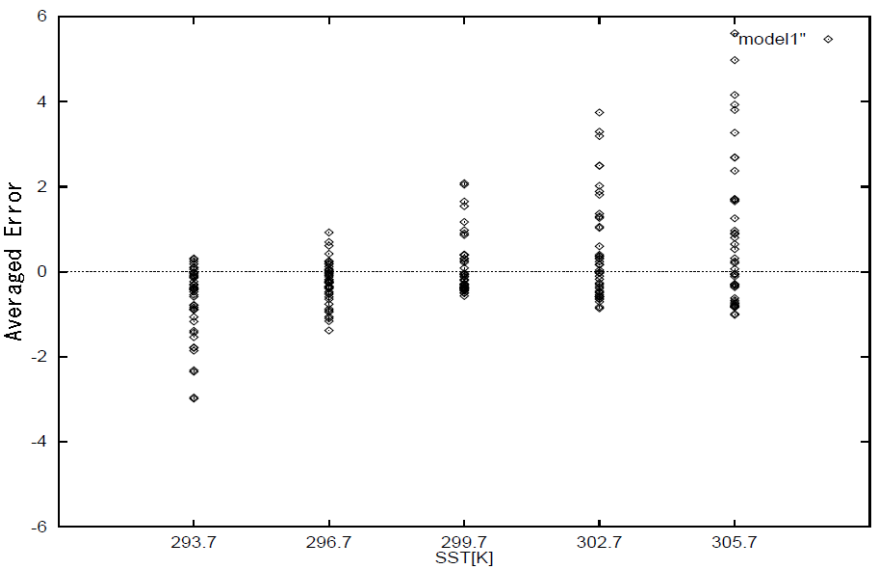

(b)Split Window

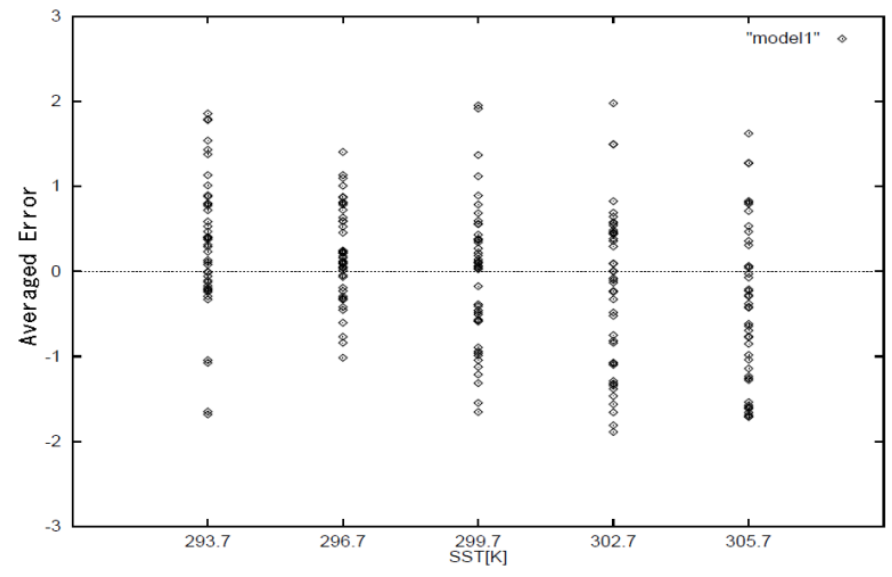

(c)Conjugate Gradient

Fig.2. Averaged SST estimation error for Tropic atmosphere model

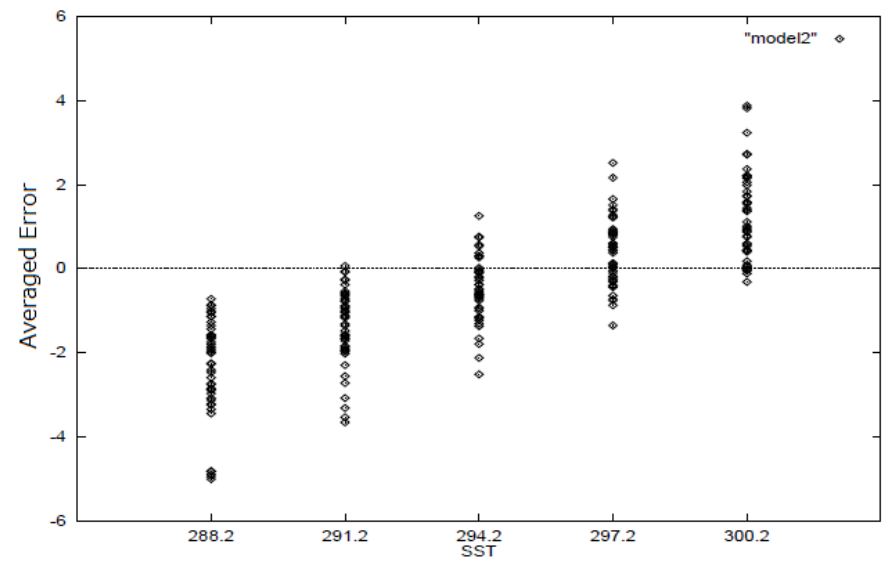

(a)MCSST 


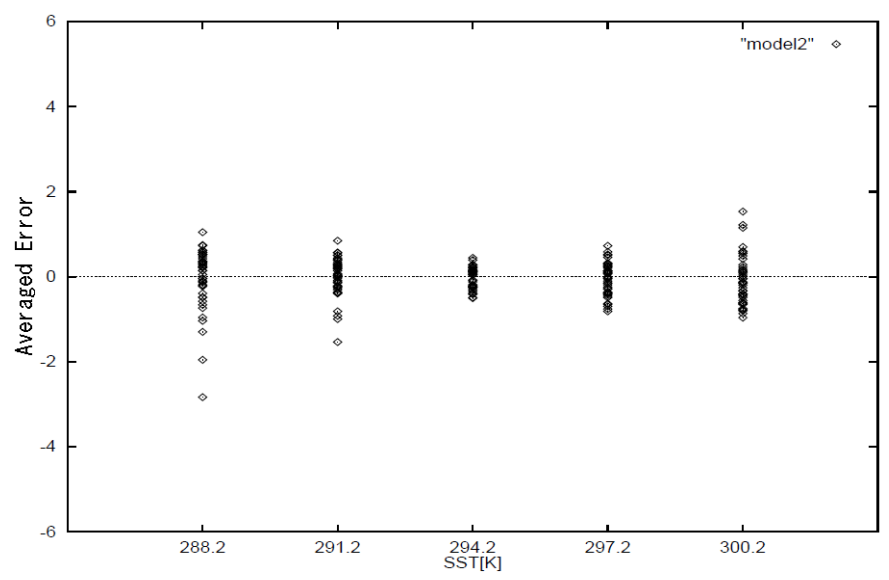

(b)Split Window

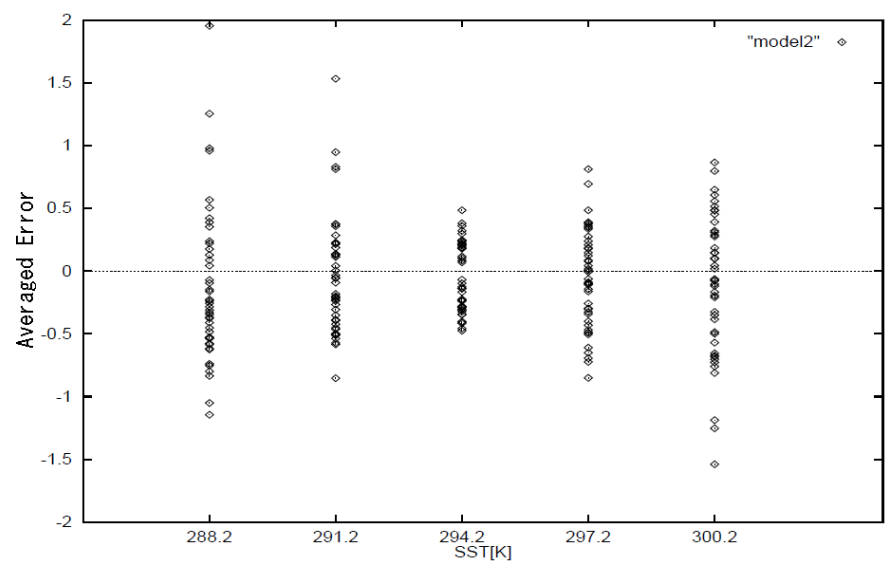

(c)Conjugate Gradient

Fig.3. Averaged SST estimation error for Mid. Latitude Summer atmosphere model

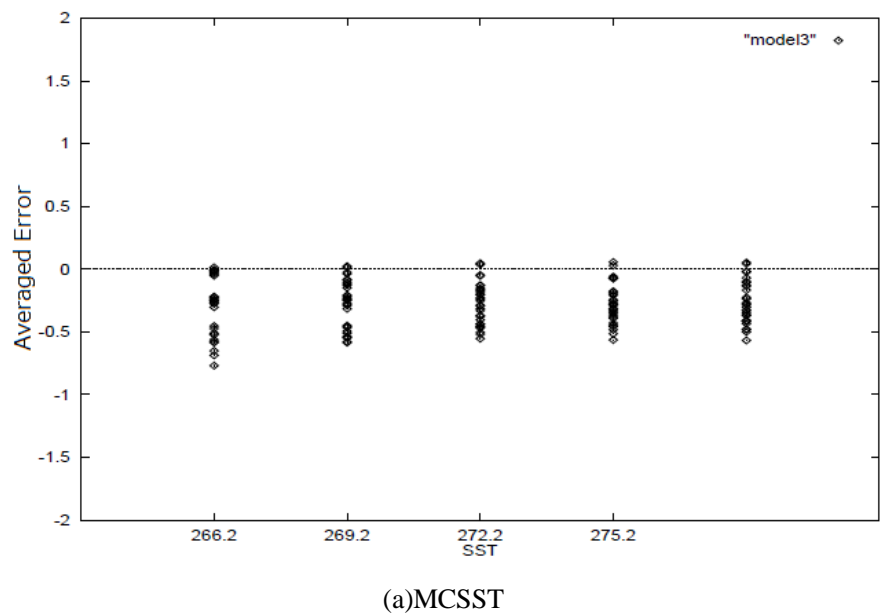

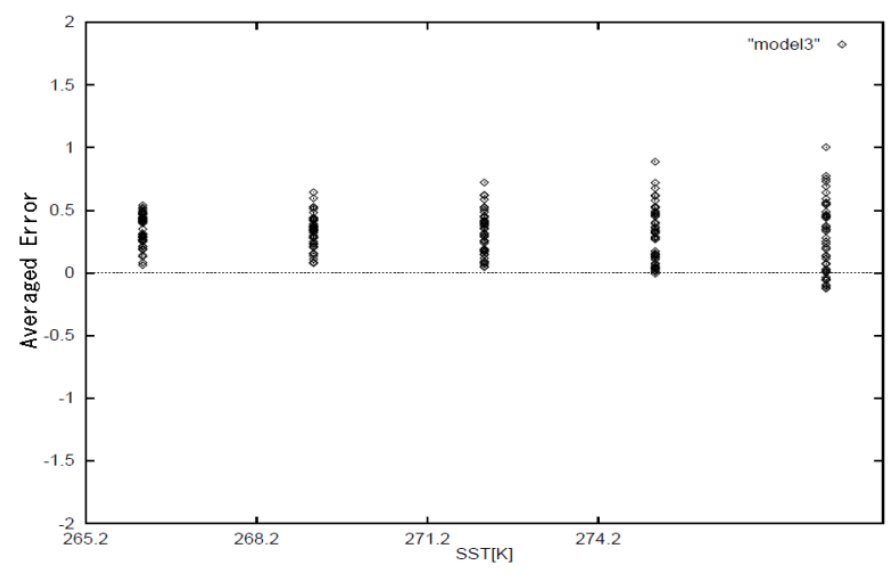

(b)Split Window

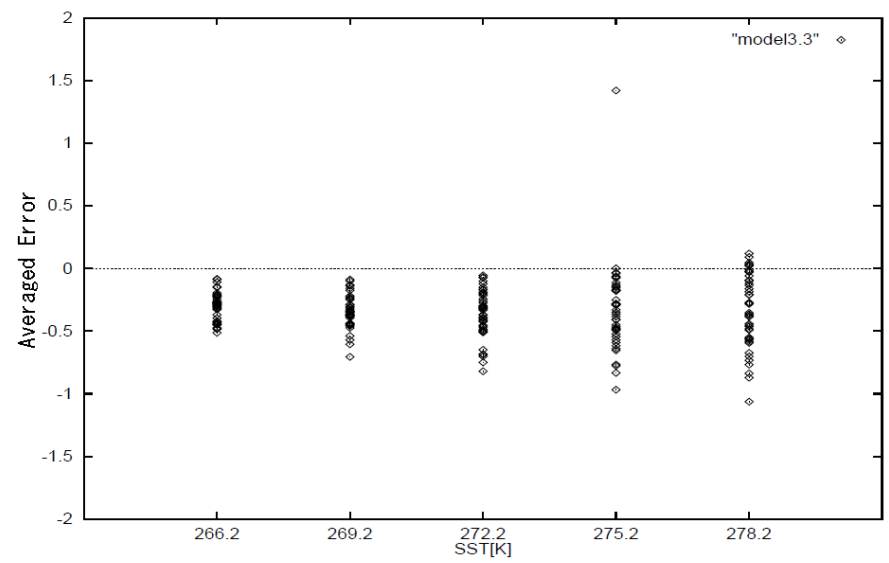

(c)Conjugate Gradient

Fig.4. Averaged SST estimation error for Mid. Latitude Winter atmosphere model

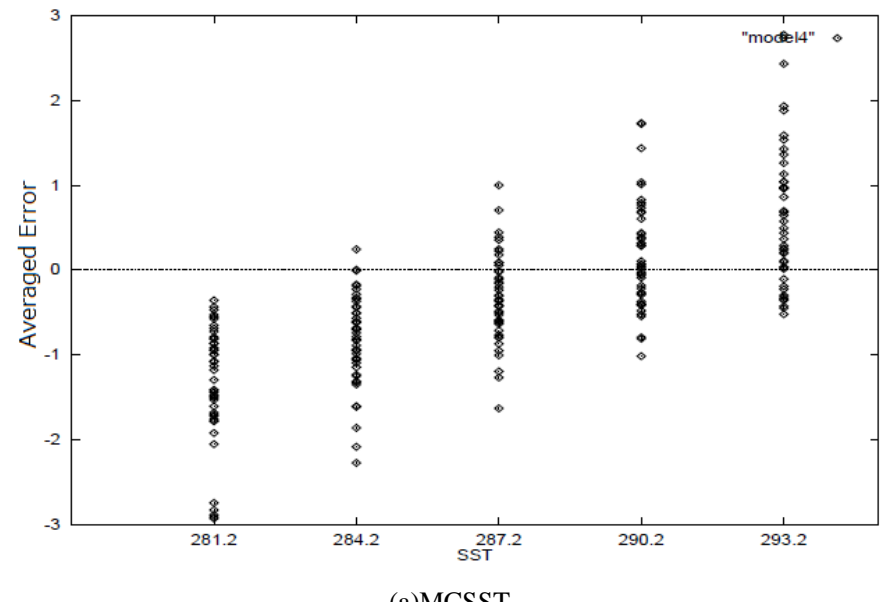

(a)MCSST 


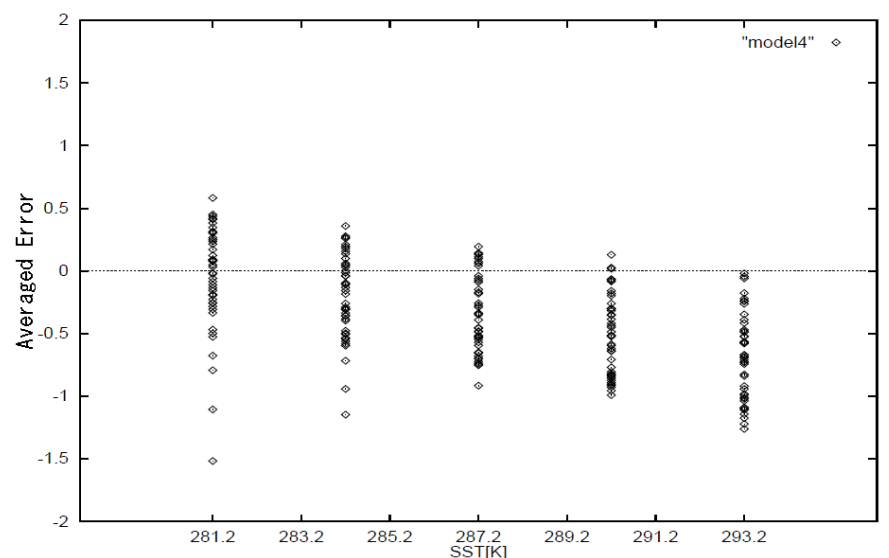

(b)Split Window

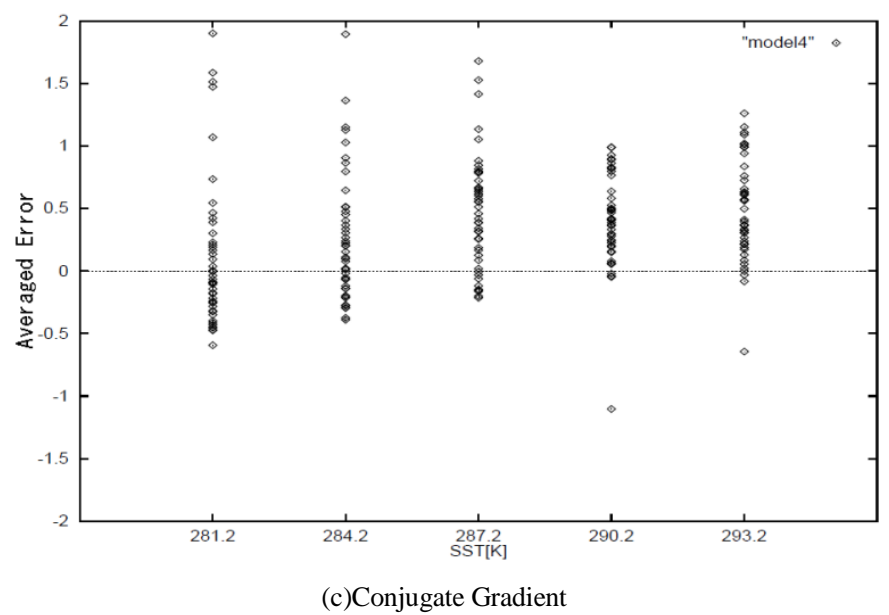

Fig.5. Averaged SST estimation error for Sub Arctic Summer atmosphere model

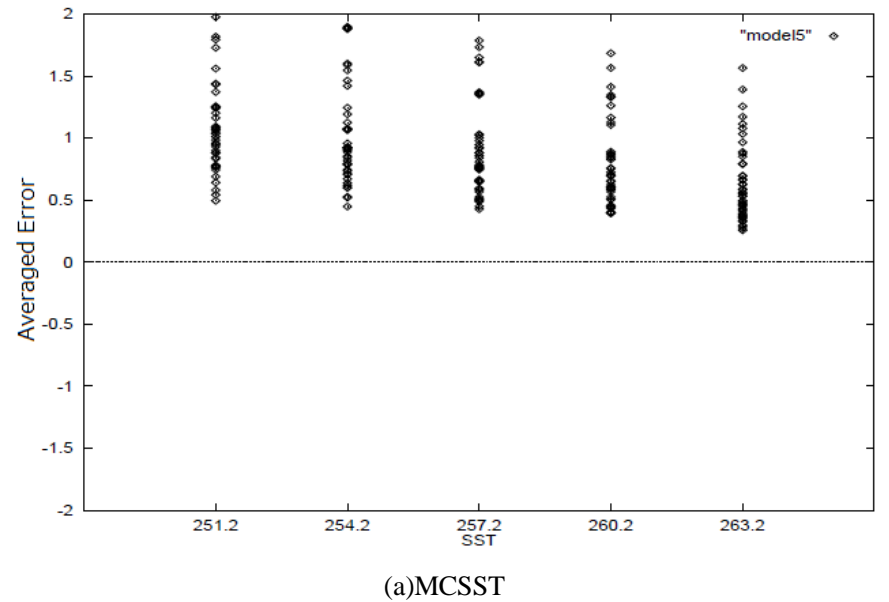

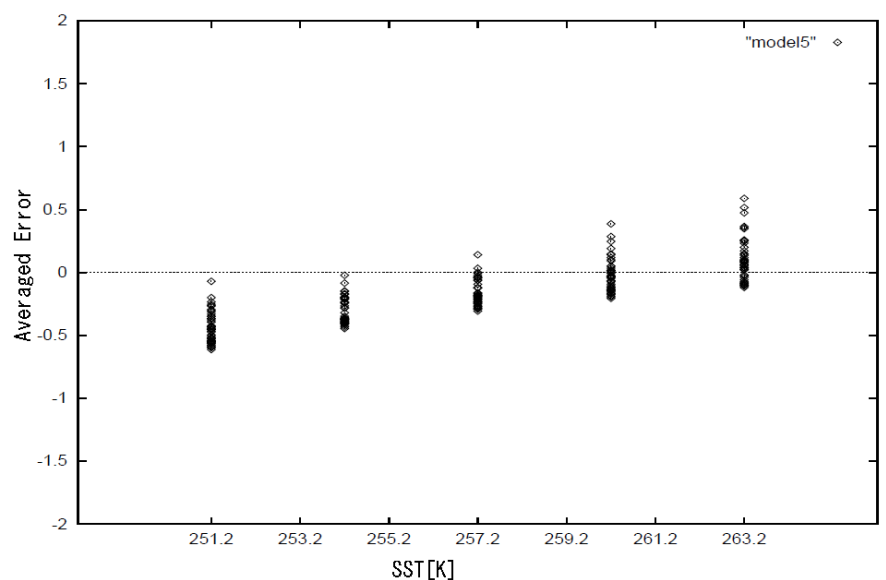

(b)Split Window

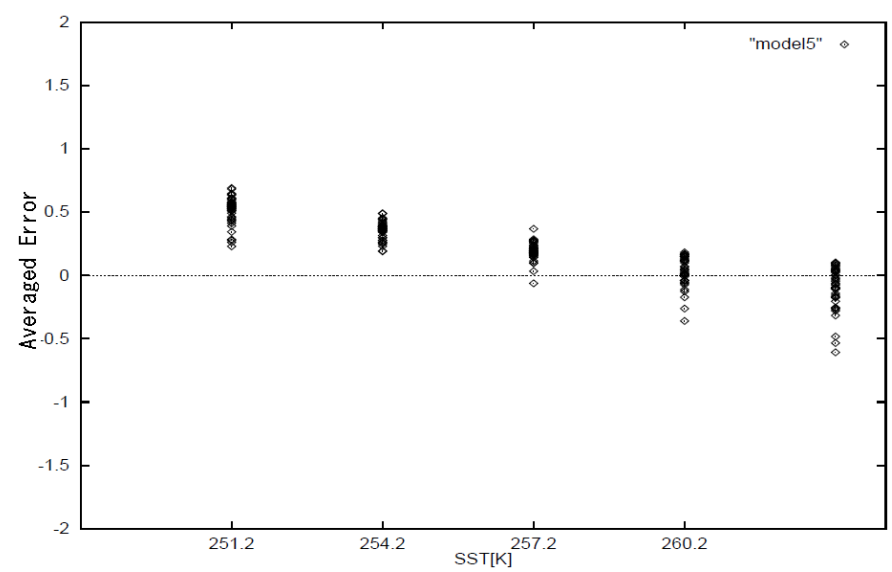

(c)Conjugate Gradient

Fig.6. Averaged SST estimation error for Sub Arctic Winter atmosphere model

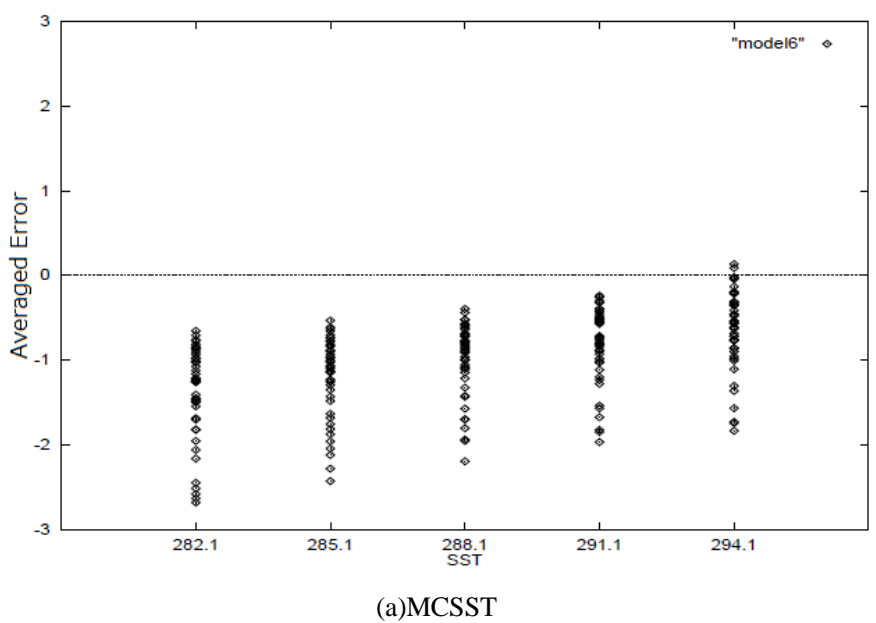




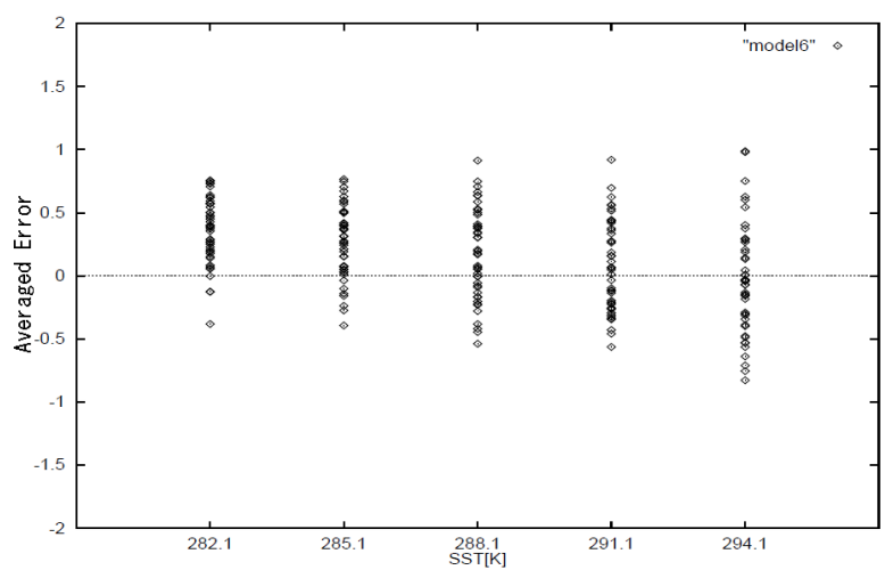

(b)Split Window

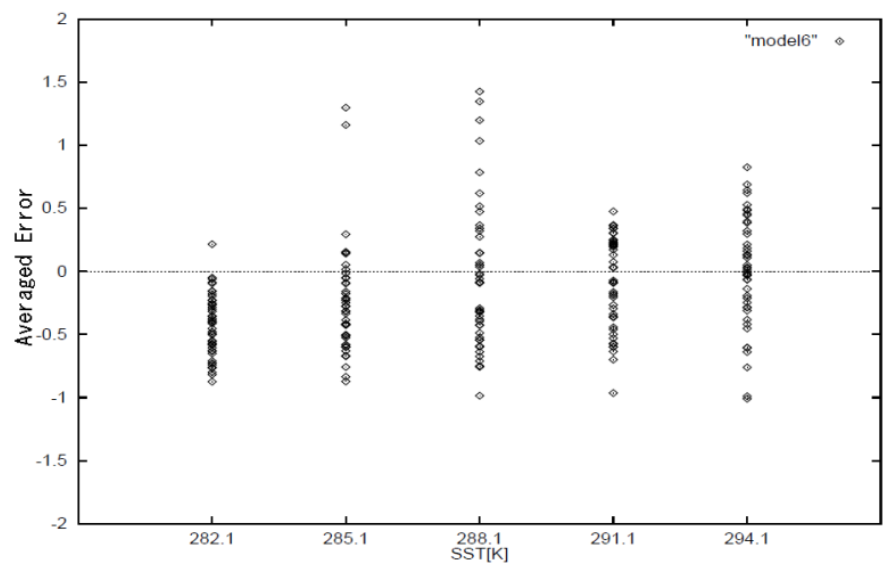

(c)Conjugate Gradient

Fig.7. Averaged SST estimation error for 1976 US standard atmosphere model

TABLE III. RMSE OF SST ESTIMATION FOR THE CONVENTIONAL MCSST, SPLIT WINDOW AND CONJUGATE GRADIENT METHOD FOR THE DIFFERENT ATMOSPHERIC MODELS

\begin{tabular}{|l|r|r|r|}
\hline Atmospheric Model & MCSST(K) & \multicolumn{1}{|l|}{$\begin{array}{l}\text { Split } \\
\text { Window(K) }\end{array}$} & CGM Method(K) \\
\hline Tropic & 2.77 & 1.195 & 0.864 \\
\hline Mid. Latitude Summer & 1.67 & 0.702 & 0.479 \\
\hline Mid. Latitude Winter & 0.72 & 0.472 & 0.408 \\
\hline Sub Arctic Summer & 1.01 & 0.641 & 0.596 \\
\hline Sub. Arctic Winter & 0.99 & 0.483 & 0.317 \\
\hline 1976 US Standard & 1.15 & 0.505 & 0.478 \\
\hline Average & 1.53 & 0.726 & 0.559 \\
\hline
\end{tabular}

\section{Evaluated Averaged SST Estimation Error}

Averaged SST estimation error of MCSST method, Split Window method, and Conjugate Gradient method for the basic atmospheric models, Tropic (Figure 2), Mid. Latitude Summer (Figure 3) and Winter (Figure 4), Sub Arctic Summer (Figure 5) and Winter (Figure 6) as well as 1976 US standard atmosphere (Figure 7).
For all cases, the proposed conjugate gradient method is superior to the other conventional methods of MCSST and Split Window, in particular for thick atmosphere (precipitable water rich atmosphere). Furthermore, MCSST has systematic errors of which the SST estimation error increases in accordance with increasing of SST.

Although only the difference between MCSST and Split Window methods is the regression coefficients, Split Window method is superior to the MCSST method because the regression coefficients are different among the atmospheric models for Split Window method; MCSST method uses the same coefficients for all atmospheric models.

\section{CONCLUSION}

Comparative study on Sea Surface Temperature: SST estimations among the conventional Multi-Channel Seat Surface Temperature: MCSST, split window method and the proposed Conjugate Gradient based method: CGM with Thermal Infrared Radiometer:

TIR data through simulations is conducted. Utilizing the proposed linearized inversion of radiative transfer equation, SST can be estimated. SST estimation accuracy of the proposed method is compared to the conventional regression based method (Split Window method and MCSST).

Through the experiments, it is found that the proposed conjugate gradient method is superior to the other conventional methods of MCSST and Split Window for all cases, in particular for thick atmosphere (precipitable water rich atmosphere).

Furthermore, MCSST has systematic errors of which the SST estimation error increases in accordance with increasing of SST. Although only the difference between MCSST and Split Window methods is the regression coefficients, Split Window method is superior to the MCSST method because the regression coefficients are different among the atmospheric models for Split Window method; MCSST method uses the same coefficients for all atmospheric models.

\section{ACKNOWLEDGMENT}

The author would like to thank Mr. Noriyuki Takamatsu for his effort to conduct the experiments.

\section{REFERENCES}

[1] Anding, D. and R. Kauth (1970): Estimation of sea surface temperature from space. Remote Sens. Environ., 1, 217-220.

[2] McMillin, L. M. (1975): Estimation of sea surface temperatures from two infrared window measurements with different absorption. $J$. Geophys. Res., 80, 5113-5117.

[3] Prabhakara, C., G. Dalu and V. G. Kunde (1974): Estimation of sea surface temperature from remote sensing in the 11 - to $13-\mu \mathrm{m}$ window region. J. Geophys. Res., 79, 5039-5044.

[4] Kohei Arai, Preliminary Assessment of Radiometric Accuracy for MOS1 Sensors, International Journal of Remote Sensing, Vol.9, No.1, pp.512, Apr.1988.

[5] Kidwell, K. B. (1991): NOAA Polar Orbiter Data Users Guide. NOAA/NESDIS/NCDC/SDSD, Washington, D.C., 192 pp.

[6] Barton, I. J. (1995): Satellite-derived sea surface temperatures: Current status. J. Geophys. Res., 100, 8777-8790. 
[7] Moriyama,M., K.Mima and K.Arai, Comparison of the sea surface temperature estimation methods, Advances in Space Reserach, Vol.16, NO.10, pp.121-124, A1-044, July 1994.

[8] K.Arai, Hidemasa Kobayashi, Masao Moriyama, Sea surface temperature estimation method based on Inversion and Linearized Inversion, Journal of Remote Sensing Society of Japan, 18, 3, 43-50, 1998 .

[9] K.Arai, Optimum band selection for sea surface teperature estimation based on regressive analysis, Journal of Japanese Remote Sensing and Photogrammetry, 39, 2, 74-81, 2000.

[10] K.Arai, Sea Surface Temperature estimation method for ocean areas and seasons using Geographic Information System as a neural network, Sediment and Ecohydraulics, Proc.in Marine Science, 9, Elsevier ISBN978-0-444 53184-1, 2007.

\section{AUTHORS PROFILE}

Kohei Arai, He received BS, MS and PhD degrees in 1972, 1974 and 1982 , respectively. He was with The Institute for Industrial Science, and Technology of the University of Tokyo from 1974 to 1978 also was with National Space Development Agency of Japan (current JAXA) from 1979 to 1990. During from 1985 to 1987, he was with Canada Centre for Remote Sensing as a Post Doctoral Fellow of National Science and Engineering Research Council of Canada. He was appointed professor at Department of Information Science, Saga University in 1990. He was appointed councilor for the Aeronautics and Space related to the Technology Committee of the Ministry of Science and Technology during from 1998 to 2000. He was also appointed councilor of Saga University from 2002 and 2003 followed by an executive councilor of the Remote Sensing Society of Japan for 2003 to 2005. He is an adjunct professor of University of Arizona, USA since 1998. He also was appointed vice chairman of the Commission "A" of ICSU/COSPAR in 2008. He wrote 30 books and published 332 journal papers. 